\title{
Parámetros poblacionales del erizo de mar Arbacia dufresnii (Arbacioida, Arbaciidae) en golfos norpatagónicos invadidos por el alga Undaria pinnatifida (Laminariales, Alariaceae)
}

\author{
Lucía Epherra ${ }^{1}$, Antonela Martelli ${ }^{2}$, Enrique M. Morsan ${ }^{3} \&$ Tamara Rubilar ${ }^{2,4}$ \\ 1. Instituto de Diversidad y Evolución Austral (IDEAus-CONICET), Puerto Madryn, Chubut, Argentina. \\ epherra@cenpat-conicet.gob.ar \\ 2. Laboratorio de Oceanografía Biológica-Centro para el Estudio de Sistemas Marinos (LOBio-CESIMAR-CONICET), \\ Puerto Madryn, Chubut, Argentina. martelli@cenpat-conicet.gob.ar, rubilar@cenpat-conicet.gob.ar \\ 3. Centro de Investigación Aplicada y Transferencia Tecnológica en Recursos Marinos "Almirante Storni”, San Antonio \\ Oeste, Río Negro, Argentina Argentina. qmorsan@gmail.com \\ 4. Universidad Nacional de la Patagonia San Juan Bosco, Puerto Madryn, Chubut, Argentina.
}

Recibido 13-I-2017. Corregido 20-VI-2017. Aceptado 16-VIII-2017.

\begin{abstract}
Population parameters of the sea urchin Arbacia dufresnii (Blainville, 1825) from North Patagonian gulfs invaded by kelp Undaria pinnatifida (Harvey) Suringar, 1873. The Asian seaweed Undaria pinnatifida invaded Argentina in 1992 (Golfo Nuevo, Patagonia). Its range has expanded and it has changed the native benthic community. Sea urchins are usually generalist herbivores that have a key role in controlling seaweeds. Arbacia dufresnii is the most abundant sea urchin in the coastal areas of northern Patagonia. The aim of this study was to relate the population parameters of $A$. dufresnii to the presence of the invasive seaweed. In 2012 we sampled sites with different invasion stages (advanced: Golfo Nuevo, recently invaded: San José Gulf, Punta Tehuelche; no invasion: San José Gulf, Zona 39). Sea urchin density was higher in the invaded sites and varied with the seaweed cycle. In addition, there were variations in the size distribution in the invaded sites. The invasive algae probably affects urchin populations because it is a new source of food that changes environmental structure (it provides microhabitat for new recruits) and affects the native benthic community. Rev. Biol. Trop. 65(Suppl. 1): S101-S112. Epub 2017 November 01.
\end{abstract}

Key words: Echinoidea; Density; Size Structure; Biological Invasions; Patagonia.

En zonas costeras, las comunidades bentónicas forman parte de uno de los eslabones más importantes en el flujo de materia y energía, siendo relevantes para la preservación de los ecosistemas marinos (Zaixso et al., 2015). Su estructura y las características poblacionales de los organismos que la componen se relacionan con la disponibilidad de recursos, las características del ambiente y la interacción entre los organismos (Sala et al., 1998; Beddingfield \& McClintock, 2000; Vadas, Barry, Beal, \& Dowling, 2002). En muchas comunidades bentónicas, los erizos de mar son los herbívoros dominantes y tienen un papel fundamental en la estructura y distribución de las asociaciones de macroalgas (Lawrence, 1975). Las poblaciones de erizos de mar pueden tolerar fluctuaciones en su densidad debido a que poseen una gran plasticidad en la asignación de recursos en los diferentes órganos (Johnson \& Mann, 1982). En diademátidos, se ha registrado una relación inversa entre la talla (diámetro del caparazón) y la densidad en muchas poblaciones (Levitan, 1989; Garrido, 2003; Clemente, Hernández, Toledo, \& Brito, 2007). Este efecto denso-dependiente evidencia la capacidad de los erizos de mar de sobrevivir a altas densidades con una talla media poblacional menor (Clemente et al., 2007). 
La dinámica poblacional en las especies pertenecientes al género Arbacia ha sido escasamente estudiada en todo el mundo (Gianguzza \& Bonaviri, 2013). Arbacia dufresnii (Blainville, 1825) es el erizo de mar más abundante en las zonas costeras del norte de la Patagonia en el Océano Atlántico (Zaixso \& Lizarralde, 2000; Morsan, 2008; Brogger et al., 2013; Bravo, Márquez, Marzinelli, Mendez, \& Bigatti, 2015). Esta especie presenta una relación inversa entre su tamaño corporal y la densidad poblacional en diferentes localidades: los erizos de mar de la población del Golfo San Jorge se encuentran en baja densidad (0.3-0.6 ind. $\left.\mathrm{m}^{-2}\right)$ y duplican el diámetro de caparazón $(52.75 \mathrm{~mm})$ de los erizos de mar de la población del Golfo Nuevo, la cual es más densa (3-10 ind. $\mathrm{m}^{-2}, 28.75 \mathrm{~mm}$ ) (Epherra et al., 2014).

Algunos sectores de la costa Argentina han sido invadidos por Undaria pinnatifida, alga nativa del continente asiático (Casas \& Piriz 1996; Martín \& Cuevas, 2006; Meretta, Matula, \& Casas, 2012; Dellatorre, Amoroso, Saravia, \& Orensanz, 2014). Este alga se registró por primera vez en Argentina en el Golfo Nuevo en 1992 (Casas \& Piriz, 1996), y en la actualidad se encuentra en un estadio de invasión avanzado (Raffo, Irigoyen, Casas, \& Schwindt, 2012). En cambio, en el Golfo San José (separado del primero por el istmo Ameghino) la presencia de $U$. pinnatifida fue reportada en 2004 (Irigoyen, 2009), y hasta 2009 solo fue registrada en las costas sur del golfo. Por lo que en la actualidad, este golfo presenta la particularidad de poseer zonas con presencia y ausencia de $U$. pinnatifida. Por otro lado, el alga invasora presenta un ciclo heteromórfico (fases microscópica y macroscópica) anual, donde la fase macroscópica (esporofítica) perdura de 6 a 9 meses (Dellatorre, Amoroso, \& Barón, 2012), es decir, las praderas de $U$. pinnatifida son estacionales. Experimentos in situ realizados en el Golfo Nuevo han demostrado que la presencia de este alga invasora está asociada con una disminución en la riqueza y diversidad de especies de algas nativas (Casas, Piriz, \& Scrosati, 2004) y un aumento en la abundancia de invertebrados marinos, entre ellos, A. dufresnii (Irigoyen, Trobbiani, Sgarlatta \& Raffo, 2011).

Undaria pinnatifida genera una perturbación en las comunidades bentónicas que invade, y probablemente produce también modificaciones en las relaciones tróficas en dichas comunidades, entre ellas la disponibilidad de alimento estacional para los erizos de mar. El objetivo de esta trabajo fue estudiar la densidad y la estructura de tallas del equinoideo nativo $A$. dufresnii en ambientes similares pero con diferentes tiempos de invasión. Este estudio busca determinar posibles interacciones entre ambas especies, explorando los rasgos ecológicos que determinan una invasión exitosa de una especie exótica y que son críticos para predecir y/o prevenir posibles efectos de futuras invasiones (Parker, Burkepile, \& Hay, 2006).

\section{MATERIALES Y MÉTODOS}

Sitio de estudio: Los golfos San José (GSJ) y Nuevo (GN) (Fig. 1), ubicados en el área costera Norte de la Patagonia Argentina, pertenecen al Área Natural Protegida de Península Valdés (Patrimonio Natural MundialUNESCO 1999). El GSJ es una pequeña cuenca semicerrada que se conecta con el Golfo San Matías por una boca angosta y poco profunda. La circulación de agua, el ingreso y egreso de la onda de marea como la topografía de la costa son responsables de un sistema frontal (Amoroso \& Gagliardini, 2010) que divide al golfo en los dominios Este y Oeste. La temperatura promedio del agua superficial varía entre $12{ }^{\circ} \mathrm{C}$ y $20{ }^{\circ} \mathrm{C}$ en el dominio este; y entre $12{ }^{\circ} \mathrm{C}$ y $18{ }^{\circ} \mathrm{C}$ en el dominio oeste (Amoroso \& Gagliardini, 2010) y la salinidad promedio es 33.82 (Zaixso et al., 1998). Por otro lado, el GN, también es una bahía semicerrada con una boca estrecha y poco profunda que conecta directamente con el Océano Atlántico. La temperatura del agua varía a lo largo del año entre $9{ }^{\circ} \mathrm{C}$ y $19{ }^{\circ} \mathrm{C}$ (Dellatorre, Pisoni, Barón \& Rivas, 2012) y la salinidad varía entre 33.5 y 33.9 (Rivas \& Ripa, 1989). 

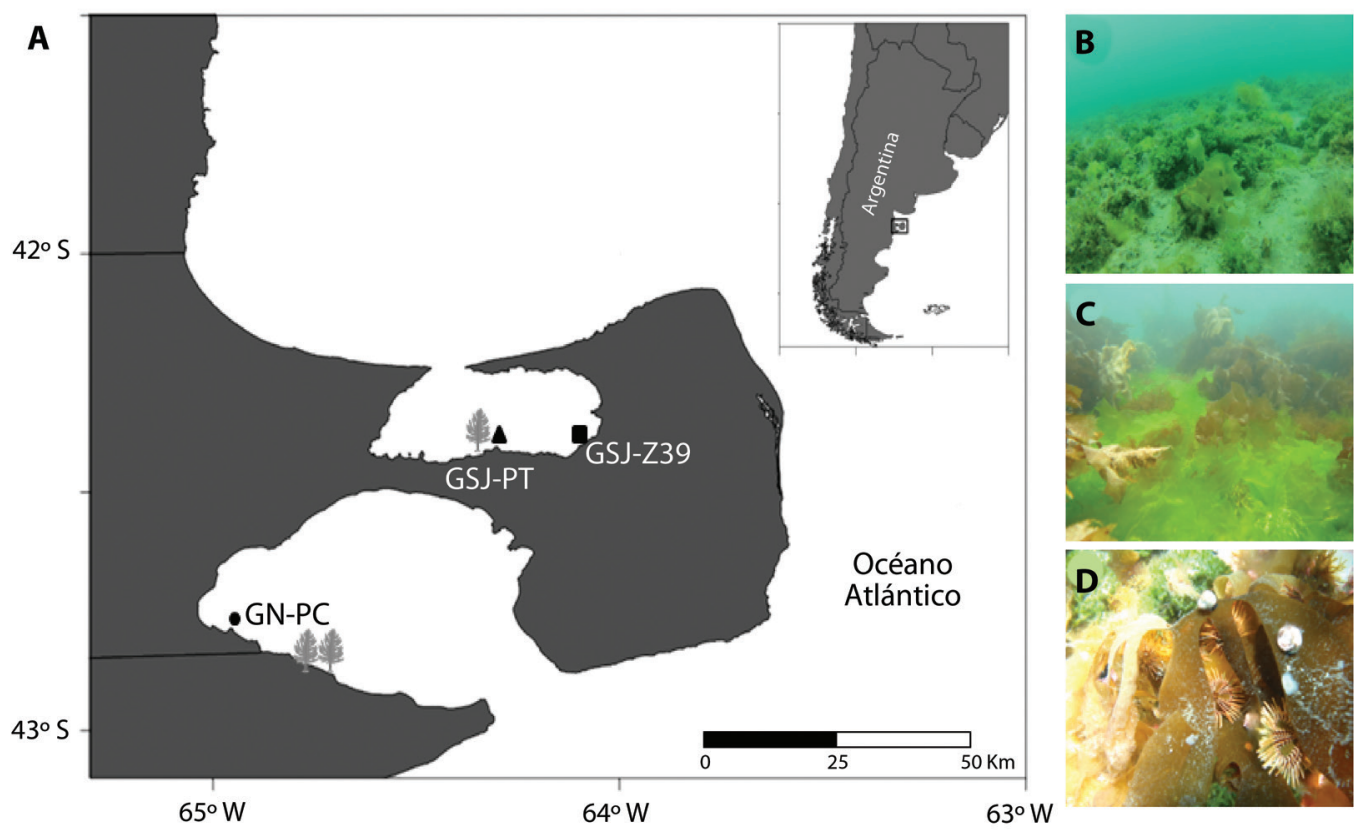

Fig. 1. a) Sitios de estudio con diferente tiempo de invasión de Undaria pinnatifida. GSJ-Z39: Golfo San José-Zona 39; GSJ-PT: Golfo San José-Punta Tehuelche y GN-PC: Golfo Nuevo-Punta Cuevas. b) Paisaje submarino de GSJ-Z39 (noviembre de 2012). c) Paisaje submarino de GN-PC (noviembre de 2012). d) Individuos de Arbacia dufresnii sobre ejemplar de Undaria pinnatifida.

Fig. 1. a) Studied sites with different invasion time of Undaria pinnatifida. GSJ-Z39: San José Gulf-Zona 39;GSJ-PT, San José Gulf-Punta Tehuelche and GN-PC, Nuevo Gulf-Punta Cuevas; b) underwater seascape of GSJ-Z39 (november 2012); c) underwater seascape of GN-PC (november 2012); d) d) Individuals of Arbacia dufresnii on Undaria pinnatifida.

Los sitios seleccionados en GSJ para este estudio fueron: Punta Tehuelche (GSJ-PT, $42^{\circ}$ $\left.23^{\prime} \mathrm{S}-64^{\circ} 17^{\prime} \mathrm{O}\right)$ que se encuentra invadido recientemente por $U$. pinnatifida; y Zona 39

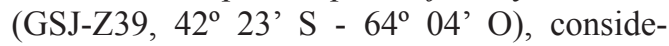
rado sitio desprovisto de dicha alga. El sitio seleccionado para el GN fue Punta Cuevas (GN-PC, 42 46' 44" S - 64' 59' 52" O), donde actualmente la invasión se encuentra en un estadio avanzado (Raffo et al., 2012). Estos sitios fueron elegidos por poseer diferente tiempo de invasión, lo cual se ve reflejado en la abundancia de $U$. pinnatifida (Cuadro 1). A su vez, estudios realizados previos a la invasión los mencionan con características biocenológicas similares (Zaixso et al., 1998; Boraso de Zaixso, Zaixso \& Casas, 1999), siendo Arbacia dufresnii es una de las especies representativas.

Muestreo: Durante el 2012 se realizaron muestreos en los tres sitios previamente descriptos. Las épocas del año se seleccionaron en

CUADRO 1

Abundancia de Undaria pinnatifida (media \pm desvío estándar; ind. $\mathrm{m}^{-2}$ ) por estación del año (2012) en sitios con diferente tiempo de invasión

TABLE 1

Undaria pinnatifida density (mean \pm standard deviation, ind. $\mathrm{m}^{-2}$ ) at annual season and at studied sites with different time of invasion

\begin{tabular}{lcccc}
\multicolumn{1}{c}{ Tiempo de invasión } & Verano & Otoño & Invierno & Primavera \\
Avanzada (GN-PC) & $4.73 \pm 2.18$ & 0 & $2.53 \pm 1.40$ & $5.99 \pm 1.73$ \\
Reciente (GSJ-PT) & $1.36 \pm 1.80$ & 0 & $0.03 \pm 0.06$ & $1.81 \pm 0.93$ \\
\hline
\end{tabular}


función del ciclo de vida de U. pinnatifida en el Golfo Nuevo (Casas, Piriz, \& Parodi, 2008): i) enero (verano) cuando el alga se encuentra en estadio de senescencia; ii) mayo (otoño) cuando los fondos están libres del alga invasora debido a que se encuentra en fase gametofítica; iii) agosto (invierno) cuando los esporofitos comienzan a crecer y; iv) noviembre (primavera) cuando los esporofitos están en su máximo desarrollo y se encuentran maduros.

En cada sitio con diferente tiempo de invasión se realizaron dos subgrupos de cuatro o cinco transectas (de $20 \mathrm{~m}$ de largo y $1 \mathrm{~m}$ de ancho, $\mathrm{N}_{\text {total }}=108$ ) perpendiculares a la costa $\mathrm{y}$ separadas entre sí por $50 \mathrm{~m}$. La recolección de muestras fue mediante buceo autónomo, donde se recolectaron manualmente todos los organismos en un salabardo (arte de pesca que consiste en un saco de red colocado en un aro de hierro) de $1 \mathrm{~cm}$ de malla. Se estimó la densidad (ind. $\mathrm{m}^{-2}$ ) de $U$. pinnatifida (Cuadro 1) y de A. dufresnii en cada transecta. La talla de los erizos de mar se obtuvo mediante la medición del diámetro del caparazón usando un calibre digital de precisión $(0.01 \mathrm{~mm}) \mathrm{y}$, de acuerdo al valor, los erizos fueron clasificados como adultos y juveniles (diámetros mayores y menores a $12 \mathrm{~mm}$, respectivamente; de acuerdo a Bernasconi, 1953).

Análisis estadístico: Las comparaciones de las densidades y de los diámetros del caparazón se analizaron utilizando el análisis de la varianza (ANOVA) realizado en la rutina PERMANOVA de Primer V6 con tiempo de invasión (TI) y estación del año (Est) como factores fijos, y los subgrupos de transectos como factores aleatorios anidados dentro del tiempo de invasión. Se utilizó la distancia euclídea y 4999 permutaciones. Cuando las diferencias fueron significativas, se analizaron comparaciones a posteriori por permutaciones (Anderson, 2004).

Las estructuras de tallas, utilizando el diámetro del caparazón, se analizaron por medio de distribuciones de frecuencia de tallas. Las distribuciones de frecuencias fueron comparadas de a pares entre sitios con la prueba de
Kolmogorov-Smirnov, utilizando el programa estadístico InfoStat (Di Rienzo et al., 2011). El software de análisis Fisat II ${ }^{\circledR}$ fue empleado para la identificación de cada componente modal en todos los sitios con diferente tiempo de invasión y estaciones excepto GSJ-Z39, debido a que el número de individuos de las muestras fue reducido.

\section{RESULTADOS}

Densidad: El rango de densidad poblacional de Arbacia dufresnii fue entre 0 y 35.75 ind. $\mathrm{m}^{-2}$ (GSJ-PT: $9.06 \pm 8.51$ ind. ${ }^{-2}$; GSJZ39: $3.94 \pm 4.49$ ind.m ${ }^{-2}$; GN-PC: $6 \pm 5.27$ ind. $\mathrm{m}^{-2}$ ). La interacción entre el tiempo de invasión y la estación del año fue significativa (Pseudo-F= 33.669, $\mathrm{p}=0.0002$ ) (Cuadro 2A). En términos generales las mayores densidades se registraron en el sitio con tiempo de invasión reciente y, en las zonas invadidas se registraron diferencias en la densidad entre las estaciones (Cuadro 2B, Fig. 2).

Estructura de tallas: El diámetro del caparazón de los 3534 individuos registrados de Arbacia dufresnii osciló entre 3.25 y $49.15 \mathrm{~mm}$. En todas las estaciones del año las tallas mayores se registraron en el sitio no invadido (GSJ-Z39; Fig. 3). En general, las distribuciones de frecuencias difirieron entre sí para cada par de estaciones del año en cada sitio (Cuadro 3).

En el sitio con invasión avanzada, el diámetro del caparazón de los erizos de mar osciló entre 3.25 y $45.02 \mathrm{~mm}$. El diámetro medio mayor se registró en primavera (noviembre) y el menor en el verano (enero) (Fig. 3c), coincidiendo con el mayor porcentaje de juveniles (Cuadro 4). En el sitio invadido recientemente, el diámetro del caparazón de los erizos de mar osciló entre 4.98 y $39.55 \mathrm{~mm}$. El $9.13 \%$ del total de individuos medidos fueron juveniles. Estos se reflejaron en la presencia de un único componente modal (Fig. 3b) el cual es mas evidente y con mayor porcentaje en la primavera (noviembre) (Cuadro 4). En el sitio no invadido, el diámetro del caparazón de los erizos 


\section{CUADRO 2}

A. Análisis de la varianza de tres vías de la comparación de densidades (ind. ${ }^{-2}$ ) de Arbacia dufresnii entre sitios con diferente tiempo de invasión (TI: avanzada, reciente y sin invadir) y estaciones del año (Est: verano, otoño, invierno y primavera). B: Comparaciones a posteriori. Los resultados significativos $(\mathrm{p}<0.05)$ se muestran en negrita

TABLE 2

A. Analysis of the three-way permutational ANOVA design, comparing Arbacia dufresnii density (ind. $\mathrm{m}^{-2}$ ).

B. Estimates for pairwise comparisons. Significant results $(\mathrm{p}<0.05)$ are shown in bold

\begin{tabular}{lccccc}
\multicolumn{1}{r}{ A-Fuente de Variación } & df & SS & MS & Pseudo-F & P(perm) \\
TI & 2 & 910.99 & 455.49 & 12.662 & 0.0316 \\
Est & 3 & 72.315 & 24.105 & 2.6355 & 0.1082 \\
Subgrupo (TI) & 3 & 108.14 & 36.047 & 2.1406 & 0.0972 \\
TIxEst & 6 & 1838.5 & 306.41 & 33.669 & $\mathbf{0 . 0 0 0 2}$ \\
EstxSubgrupo (TI) & 9 & 81.474 & 9.0526 & 0.53758 & 0.8496 \\
Residual & 81 & 1364 & 16.84 & & \\
Total & 104 & 4272.9 & & & \\
\hline
\end{tabular}

B-Comparaciones a posteriori

\begin{tabular}{|c|c|c|c|}
\hline Est & TI & $\mathrm{t}$ & $\mathrm{P}($ perm $)$ \\
\hline \multirow[t]{3}{*}{ Verano } & Avanzada-Reciente & 3.7864 & 0.0564 \\
\hline & Avanzada-Sin Invasión & 3.2964 & 0.0732 \\
\hline & Reciente- Sin Invasión & 0.6176 & 0.5946 \\
\hline \multirow[t]{3}{*}{ Otoño } & Avanzada-Reciente & 4.4797 & 0.0402 \\
\hline & Avanzada-Sin Invasión & 0.2395 & 0.8524 \\
\hline & Reciente- Sin Invasión & 1.448 & 0.2788 \\
\hline \multirow{3}{*}{ Invierno } & Avanzada-Reciente & 1.1916 & 0.3568 \\
\hline & Avanzada-Sin Invasión & 3.1372 & 0.0788 \\
\hline & Reciente- Sin Invasión & 3.2721 & 0.0580 \\
\hline \multirow[t]{3}{*}{ Primavera } & Avanzada-Reciente & 32.002 & 0.0002 \\
\hline & Avanzada-Sin Invasión & 0.3201 & 0.7782 \\
\hline & Reciente- Sin Invasión & 19.012 & 0.0002 \\
\hline TI & Est & $\mathrm{t}$ & $\mathrm{P}($ perm $)$ \\
\hline Invasión & Verano-Otoño & 2.4709 & 0.2338 \\
\hline \multirow[t]{5}{*}{ Avanzada } & Verano-Invierno & 1.2748 & 0.3958 \\
\hline & Verano-Primavera & 5.0611 & 0.1216 \\
\hline & Otoño-Invierno & 7.4038 & 0.091 \\
\hline & Otoño-Primavera & 2.488 & 0.2488 \\
\hline & Invierno-primavera & 15.537 & 0.0396 \\
\hline Invasión & Verano-Otoño & 32.007 & 0.0182 \\
\hline \multirow[t]{5}{*}{ Reciente } & Verano-Invierno & 5.0201 & 0.1176 \\
\hline & Verano-Primavera & 43.526 & 0.0152 \\
\hline & Otoño-Invierno & 0.1414 & 0.9114 \\
\hline & Otoño-Primavera & 17.494 & 0.0380 \\
\hline & Invierno-primavera & 12.119 & 0.0618 \\
\hline \multirow[t]{6}{*}{ Sin Invasión } & Verano-Otoño & 0.8683 & 0.5322 \\
\hline & Verano-Invierno & 0.8308 & 0.556 \\
\hline & Verano-Primavera & 3.1958 & 0.2032 \\
\hline & Otoño-Invierno & 1.9019 & 0.2912 \\
\hline & Otoño-Primavera & 1.307 & 0.3988 \\
\hline & Invierno-primavera & 0.7009 & 0.6156 \\
\hline
\end{tabular}




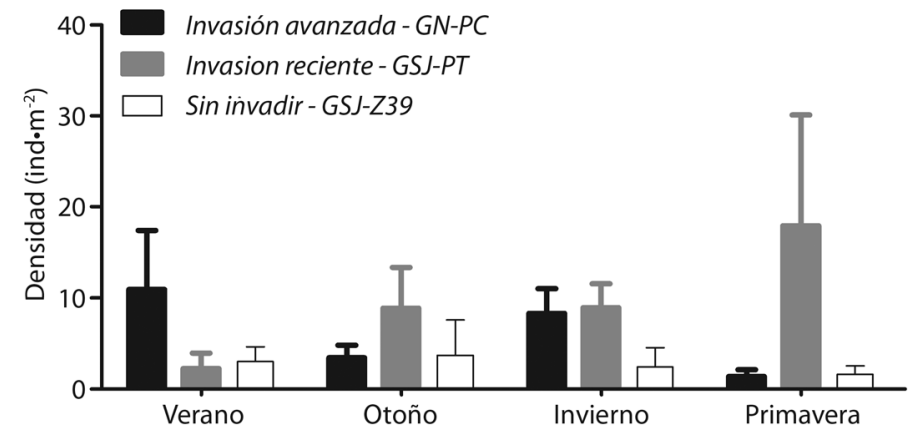

Fig. 2. Densidad Arbacia dufresnii (media \pm desvío estándar; ind.m-2) por estación del año. GN-PC: Golfo Nuevo, Punta Cuevas; GSJ-PT: Golfo San José, Punta Tehuelche; GSJ-Z39: Golfo San José, Zona 39.

Fig. 2. Arbacia dufresnii density (mean \pm standard deviation, ind. $\mathrm{m}^{-2}$ ) at annual season and at studied sites with different time of invasion.

\section{CUADRO 3}

Comparación de las distribuciones de frecuencias del diámetro del caparazón de Arbacia dufresnii entre estaciones (prueba de Kolmogorov-Smirnov) en sitios con diferente tiempo de invasión

TABLE 3

Comparison between seasons of frequency distributions of Arbacia dufresnii test diameter (Kolmogorov-Smirnov test) at sites with different time of invasion

\begin{tabular}{|c|c|c|c|c|c|c|c|}
\hline & & Ver/Oto & Ver/Inv & Ver /Prim & Oto / Inv & Oto / Prim & Inv / Prim \\
\hline \multirow[t]{2}{*}{ Invasión Avanzada } & K-S & 0.22 & 0.24 & 0.52 & 0.07 & 0.33 & 0.33 \\
\hline & $\mathrm{p}$ & $<0.01$ & $<0.01$ & $<0.01$ & $>0.05$ & $<0.01$ & $<0.01$ \\
\hline \multirow[t]{2}{*}{ Invasión Reciente } & K-S & 0.19 & 0.18 & 0.54 & 0.35 & 0.4 & 0.7 \\
\hline & $\mathrm{p}$ & $<0.01$ & $<0.01$ & $<0.01$ & $<0.01$ & $<0.01$ & $<0.01$ \\
\hline \multirow[t]{2}{*}{ Sin Invadir } & $\mathrm{K}-\mathrm{S}$ & 0.12 & 0.18 & 0.41 & 0.21 & 0.44 & 0.36 \\
\hline & $\mathrm{p}$ & $>0.05$ & $<0.05$ & $<0.01$ & $<0.01$ & $<0.01$ & $<0.01$ \\
\hline
\end{tabular}

Ver: verano; Oto: otoño; Inv: invierno; Prim: primavera.

\section{CUADRO 4}

Porcentaje de juveniles de Arbacia dufresnii por estación del año (2012) en sitios con diferente tiempo de invasión)

TABLE 4

Percentage of juveniles of Arbacia dufresnii at annual season and at studied sites with different time of invasion

\begin{tabular}{lccccc}
\multicolumn{1}{c}{ Tiempo de invasión } & $\mathrm{N}$ & Verano & Otoño & Invierno & Primavera \\
Sin Invadir (GSJ-Z39) & 525 & 0.68 & 0.58 & 0 & 0 \\
Reciente (GSJ-PT) & 1523 & 6.15 & 5.16 & 1.03 & 15.52 \\
Avanzada (GN-PC) & 1486 & 5.47 & 2.05 & 2.91 & 3.42 \\
\hline
\end{tabular}

GSJ-Z39: Zona 39, Golfo San José. GSJ-PT: Punta Tehuelche, Golfo San José. GN-PC: Punta Cuevas, Golfo Nuevo. 


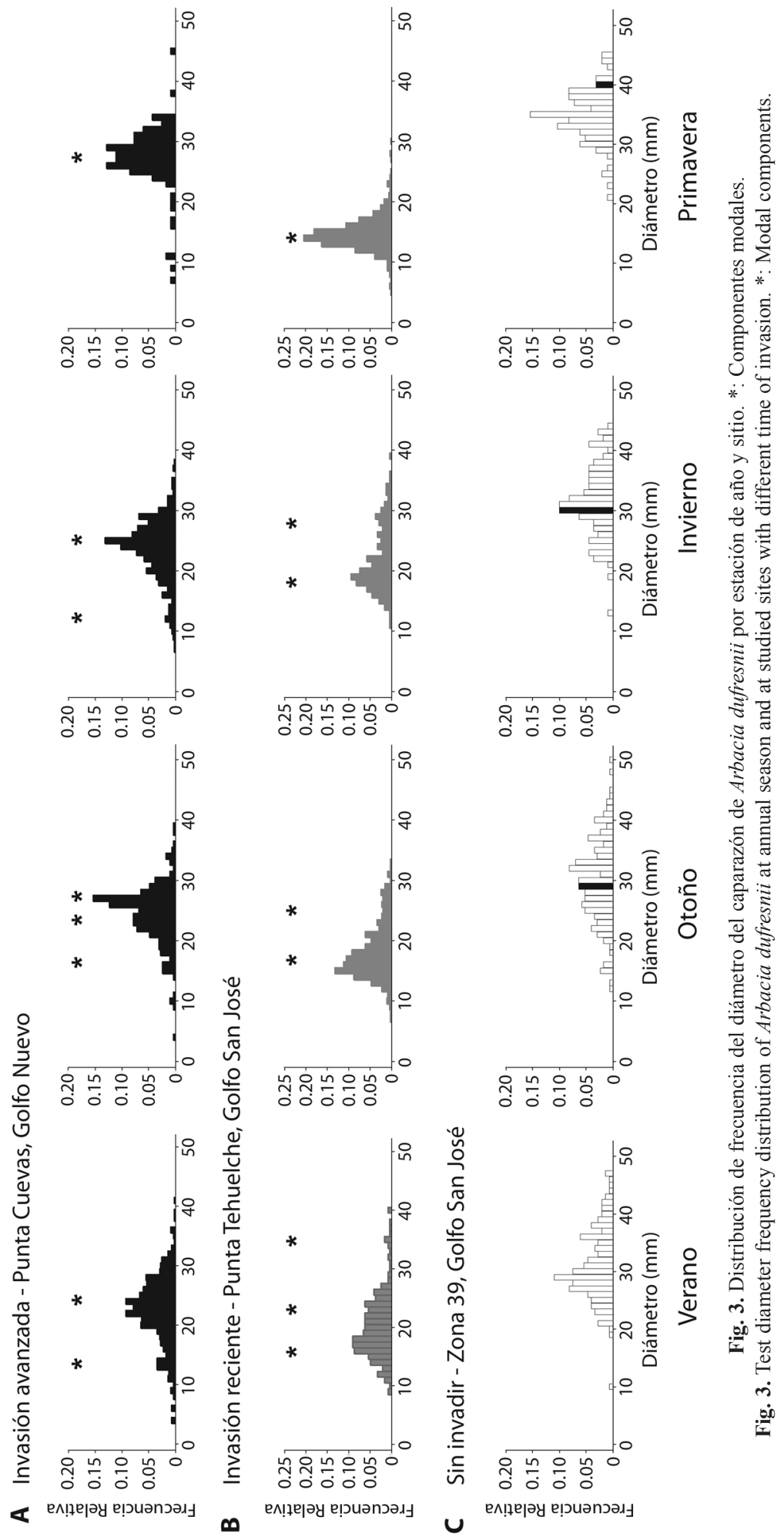


de mar osciló entre 9.63 y 49.15 mm, y sólo $0.38 \%$ del total de individuos fueron juveniles.

\section{DISCUSIÓN}

Arbacia dufresnii presentó variaciones tanto en la densidad como en la distribución de tallas en los golfos estudiados. Las variaciones pueden deberse a la complejidad del ambiente que suele afectar la abundancia de erizos de mar (Clemente \& Hernández, 2008; LabbéBellas, Cordeiro, Floeter, \& Segal, 2016). Por otro lado, las praderas del alga invasora Undaria pinnatifida son componentes altamente productivos de los ecosistemas marinos y sostienen diversas asociaciones de fauna (Ohmo, 1996) que podrían incrementar la densidad de invertebrados. Este último aspecto, ha sido descrito en experimentos de exclusión/inclusión de $U$. pinnatifida, donde los tratamientos con presencia del alga invasora mostraron una mayor riqueza y abundancia de invertebrados bentónicos, entre ellos la abundancia de $A$. dufresnii la cual se triplicó (Irigoyen et al., 2011).

El rango de densidad de $A$. dufresnii $(0 \mathrm{y}$ 35.75 ind. $\mathrm{m}^{-2}$ ) en todos los sitios estudiados resultó mayor al registrado en la región Magallánica, al sur de Chile, donde varía entre 0-5.5 ind $\mathrm{m}^{-2}$ (promedio 3 ind. $\mathrm{m}^{-2}$ ) (Newcombe, Cardenas, \& Geange, 2012). Si bien las densidades estimadas en el presente estudio son inferiores a las halladas en frentes de barrens, son relevantes debido a que pueden tener un efecto significativo sobre comunidades de macroalgas contribuyendo a regular su biomasa, como sucede en otras regiones (Palacín, Giribet, Carner, Dantart, \& Turon, 1998; Johnson, Valentine, \& Pederson, 2004; Paredes, 2010; Bonaviri, Vega Fernández, Fanelli, Badalamenti, \& Gianguzza, 2011; Newcombe et al., 2012).

En el presente trabajo se observó una tendencia en la abundancia del erizo de mar, la cual es mayor en los sitios invadidos por $U$. pinnatifida. Trabajos previos en el género Arbacia han descrito variaciones en la densidad de erizos de mar en distintos ambientes relacionados con la disponibilidad y calidad del alimento, la exposición a depredadores o a diferentes eventos de reclutamiento (Sala et al., 1998; Hill \& Lawrence, 2003; Hereu et al., 2012). En los golfos estudiados, A. dufresnii se alimenta de $U$. pinnatifida en estado senescente (Teso, Bigatti, Casas, Piriz, \& Penchaszadeh, 2009; Castro, 2014), por lo que las variaciones entre ambientes podrían relacionarse a la disponibilidad de alimento siendo el alga invasora una nueva fuente de alimento estacional. Además de las variaciones en las densidades, se registraron variaciones en las tallas de $A$. dufresnii en los sitios estudiados. En muchas especies de erizos de mar la variación de la densidad está acompañada de una variación en la talla, debido a un efecto denso-dependiente (Levitan, 1988, 1989; Brey, Pearse, Basch, Mc Clintock, \& Slattery, 1995; Hill \& Lawrence, 2003; Muthiga \& Jaccarini, 2005; BenítezVillalobos, Domínguez, \& López-Pérez, 2008; Paredes, 2010, entre otros), donde el aumento de la densidad poblacional posiblemente produzca un aumento en la competencia intraespecífica por el alimento (Garrido, 2003). Estudios previos han demostrado que en áreas invadidas por $U$. pinnatifida, A. dufresnii presenta un mayor tamaño de la linterna de Aristóteles, lo cual suele estar relacionado con indicar una menor disponibilidad de alimento por individuo (Epherra et al., 2015). Por lo tanto, las mayores abundancias de erizos de mar podrían estar afectando el crecimiento debido a una limitante en el alimento ocasionado por la reducción en biomasa y diversidad de las algas nativas (Casas et al., 2004) o porque la oferta del alimento U. pinnatifida es temporal.

Por otro lado, la estabilidad de factores ambientales y la disponibilidad de alimento en diferentes hábitats influye tanto en la densidad y sus fluctuaciones, como en la estructura poblacional de los erizos de mar (Brey et al., 1995; Turon, Giribet, Lopez, \& Palacín, 1995; Herrero-Pérezrul, Ramírez-Ortíz, RosalesEstrada, \& Reyes-Bonilla, 2015). En ambientes estables, la distribución de tallas es más uniforme a lo largo del tiempo y la densidad es menos fluctuante que en ambientes inestables (Turon et al., 1995). De esta forma, el sitio no invadido por $U$. pinnatifida (GSJ-Z39) 
podría ser un ambiente más estable respecto a los sitios invadidos, debido a que no sufre variaciones estructurales en el ambiente por la presencia del alga invasora, dando lugar a una distribución de tallas y a densidades de erizos de mar más uniformes a lo largo del tiempo. La variabilidad en la estructura de tallas encontrada en este estudio estaría influenciada por el efecto conjunto del crecimiento y la incorporación de nuevos individuos a la población adulta de los erizos de mar. En el género Arbacia, los reclutamientos son escasamente reportados (Gianguzza \& Bonaviri, 2013), debido probablemente a la existencia de una segregación de hábitats entre adultos y juveniles (Kroh, Madeira, \& Haring, 2011). Probablemente los juveniles de $A$. dufresnii podrían utilizar un ambiente diferente a los adultos en GSJ-Z39, como sucede en A. lixula (Sala et al., 1998). En cambio, en los sitios invadidos (GN-PC y GSJ-PT), se registró la incorporación de individuos juveniles. La presencia de bosques de macroalgas puede afectar las escalas espaciales de reclutamiento mediante la alteración de los patrones locales de circulación (Tegner \& Dayton, 1981), y también porque puede proporcionar superficie para el asentamiento o protección a los reclutas (Tegner \& Dayton, 1981; Pearse \& Hines, 1987; Thiel \& Vásquez, 2000; Rios, Mutschke, \& Cariceo, 2003). En los sitios estudiados, cuando el esporofilo de $U$. pinnatifida se encuentra desarrollado (primavera y verano) se encontraron individuos juveniles de A. dufresnii, así como otras especies, en dicha estructura (observación personal). Por lo tanto, es probable que la forma y tamaño de praderas de $U$. pinnatifida contribuyan a la complejidad estructural del hábitat generando un refugio para los nuevos individuos de la población y, de esta manera, favorecen la presencia de individuos juveniles en el mismo ambiente que los adultos.

En el sitio no invadido, GSJ-Z39, se registró la presencia de $U$. pinnatifida únicamente en el mes de noviembre y su biomasa fue escasa $\left(<100\right.$ g.m $\left.{ }^{-2}\right)$. Dellatorre et al. (2012a) registraron el alga invasora por primera vez en la temporada 2010-2011. De esta forma, puede inferirse que en el muestreo realizado, el alga se encontraría en el tiempo de retraso de invasión, este es definido como el tiempo que existe entre el evento de introducción y la subsecuente explosión poblacional de la especie invasora, con una baja abundancia o un rango de distribución acotado (Crooks \& Soulé, 1999). Por lo tanto, conocer la información previa a una invasión es esencial a la hora de entender los posibles efectos de una especie invasora, así como también generar medidas de manejo en áreas protegidas.

\section{AGRADECIMIENTO}

Agradecemos a los buzos R. Vera, N. Ortiz, M. Arenas, R. Passarotti y J. Natale por la ayuda otorgada en el campo. A la Administración de Áreas Protegidas de la Pcia. del Chubut por otorgar los permisos para acceder a los sitios dentro del Área Natural Protegida Península Valdés. Al Dr. José Carlos Hernández por su colaboración en la sección estadística y el acceso al programa estadístico PRIMER V6. Este trabajo forma parte de la Tesis Doctoral de la L. Epherra.

\section{RESUMEN}

Undaria pinnatifida, es un alga que ha invadido la costa de Argentina hace más de 20 años y presenta diferentes tiempos de invasión en distintos sitios. Dado que los erizos de mar son herbívoros generalistas que suelen tener un papel relevante en la resistencia a la invasión de algas, y que Arbacia dufresnii es el erizo de mar más abundante en las zonas costeras del norte de la Patagonia, el objetivo de esta trabajo fue determinar la relación entre parámetros poblacionales de este equinoideo con la presencia del alga invasora. Durante el 2012 se realizaron muestreos estacionales (enero, mayo, agosto y noviembre), de acuerdo al ciclo de vida del alga, en sitios que presentaban una invasión avanzada de $U$. pinnatifida (Golfo Nuevo), una invasión reciente (Golfo San José, Punta Tehuelche), y un sitio sin invasión (Golfo San José, Zona 39). Los sitios invadidos por $U$. pinnatifida presentaron variaciones de densidad de $A$. dufresnii, lo cual fue acompañado por variaciones en la distribución de tallas. De esta forma, bajo la hipótesis de que la disponibilidad de alimento influye en el tamaño corporal y densidad poblacional de los erizos de mar, las praderas estacionales de $U$. pinnatifida parecen estar afectando los parámetros poblacionales de A. dufresnii. 
A su vez, la superficie proporcionada por el alga invasora en determinadas épocas del año permitiría la incorporación de nuevos individuos a la población.

Palabras claves: Echinoidea; Densidad; Estructura de tallas; Invasiones biológicas; Patagonia.

\section{REFERENCIAS}

Amoroso, R. O., \& Gagliardini, D. A. (2010). Inferring complex hydrographic processes using RemoteSensed Images: Turbulent fluxes in the Patagonian Gulfs and implications for scallop metapopulation dynamics. Journal of Coastal Research, 26, 320-332.

Anderson, M. J. (2004). PERMANOVA 2factor: a FORTRAN computer program for permutational multivariate analysis of variance using permutation tests. University of Auckland, Auckland.

Beddingfield, S. D., \& McClintock, J. B. (2000). Demographic characteristics of Lytechinus variegatus (Echinodermata) from three habitats in a North Florida Bay, Gulf of Mexico. Marine Ecology, 21(1), 17-40.

Benítez-Villalobos, F., Domínguez, M., \& López-Pérez, R. (2008). Temporal variation of the sea urchin Diadema mexicanum population density at Bahias de Huatulco, Western Mexico. Revista de Biología Tropical, $56(3), 255-263$.

Bernasconi, I. (1953). Monografía de los Equinoideos Argentinos. Anales Museo Historia Natural, 6(2), 57.

Bonaviri, C., Vega Fernández, T., Fanelli, G., Badalamenti, F., \& Gianguzza, P. (2011). Leading role of the sea urchin Arbacia lixula in maintaining the barren state in southwestern Mediterranean. Marine Biology, 158(11), 2505-2513.

Boraso de Zaixso, A. L., Zaixso, H. E., \& Casas, G. (1999). Asociaciones de algas bentónicas submareales en el golfo San José (Chubut, Argentina). Physis 57(a) (132-133), 17-27.

Bravo, G., Márquez, F., Marzinelli, E. M., Mendez, M. M., \& Bigatti, G. (2015). Effect of recreational diving on Patagonian rocky reefs. Marine Environmental Research, 104(0), 31-36.

Brey, T., Pearse, J. S., Basch, L., Mc Clintock, J. B., \& Slattery, M. (1995). Growth and production of Sterechinus neumayeri (Echinoidea: Echinodermata) in McMurdo Sound, Antarctica. Marine Biology, 124, 279-292.

Brogger, M., Gil, D. G., Rubilar, T., Martinez, M., Díaz de Vivar, M. E., Escolar, M., .. . Tablado, A. (2013). Echinoderms from Argentina: Biodiversity, distribution and current state of knowledge. In J. J. Alvarado \& F. A. Solís Marin (Eds.), Latin American Echinoderms (pp. 359-402). Berlin Heidelberg: Springer .
Casas, G. N., \& Piriz, M. L. (1996). Surveys of Undaria pinnatifida (Laminariales, Phaeophyta) in Golfo Nuevo, Argentina. Hidrobiologia, 326-327, 213-215.

Casas, G. N., Piriz, M. L., \& Parodi, E. R. (2008). Population features of the invasive kelp Undaria pinnatifida (Phaeophyceae: Laminariales) in Nuevo Gulf (Patagonia, Argentina). Journal of the Marine Biological Association of the United Kingdom, 88(1), 21-28.

Casas, G. N., Piriz, M. L., \& Scrosati, R. (2004). The invasive kelp Undaria pinnatifida (Phaeophyceae, Laminariales) reduces native seaweed diversity in Nuevo Gulf (Patagonia, Argentina). Biological Invasions, 6, 411-416.

Castro, K. L. (2014). Dieta del erizo verde de mar Arbacia dufresnii y su relación con el alga invasora Undaria pinnatifida en costas del Golfo San José, Patagonia (Tesis). Universidad Nacional del Comahue, Bariloche, Argentina.

Clemente, S., \& Hernández, J. C. (2008). Influence of wave exposure and habitat complexity in determining spatial variation of the sea urchin Diadema aff. antillarum (Echinoidea: Diadematidae) populations and macroalgal cover (Canary Islands-Eastern Atlantic Ocean). Revista de Biología Tropical, 56(3), 229-254.

Clemente, S., Hernández, J. C., Toledo, K., \& Brito, A. (2007). Predation upon Diadema aff. antillarum in barren grounds in the Canary Islands. Scientia Marina, 71(4), 745-754.

Crooks, J. A., \& Soulé, M. E. (1999). Lag times in population explosions of invasive species: Causes and implications. In O. T. Sandlund, P. J. Schei \& A. Viken (Eds.), Invasive Species and Biodiversity Management (pp. 103-125). The Netherlands: Kluwer Academic Publishers.

Dellatorre, F., Amoroso, R., \& Barón, P. (2012a). El alga exótica Undaria pinnatifida en Argentina: Biología, distribución y potenciales impactos. Editorial Académica Española.

Dellatorre, F. G., Pisoni, J. P., Barón, P. J., \& Rivas, A. L. (2012b). Tide and wind forced nearshore dynamics in Nuevo Gulf (Northern Patagonia, Argentina): Potential implications for cross-shore transport. Journal of Marine Systems, 96, 82-89.

Dellatorre, F., Amoroso, R., Saravia, J., \& Orensanz, J. M. (2014). Rapid expansion and potential range of the invasive kelp Undaria pinnatifida in the Southwest Atlantic. Aquatic Invasions, 9(4), 467-478.

Di Rienzo, J. A., Casanoves, F., Balzarini, M. G., Gonzalez, L., Tablada, M., \& Robledo, C. W. (2011). InfoStat.: Grupo InfoStat.

Epherra, L., Crespi-Abril, A., Meretta, P. E., Cledón, M., Morsan, E. M., \& Rubilar, T. (2015). Morphological plasticity in the Aristotle's lantern of Arbacia 
dufresnii (Phymosomatoida: Arbaciidae) off the Patagonian coast. Revista Biologia Tropical, 63(2), 339-351.

Epherra, L., Gil, D. G., Rubilar, T., Perez-Gallo, S., Reartes, M. B., \& Tolosano, J. A. (2014). Temporal and spatial differences in the reproductive biology of the sea urchin Arbacia dufresnii. Marine and Freshwater Research, 66, 329-342.

Garrido, M. J. (2003). Contribución al conocimiento de Diadema antillarum Philippi 1845, en Canarias. PhD, Universidad de las Palmas de Gran Canaria, Las Palmas de Gran Canaria.

Gianguzza, P., \& Bonaviri, C. (2013). Arbacia. In J. M. Lawrence (Ed.), Sea urchins: Biology and Ecology (3 ed., pp. 275-283). San Diego: Elsevier Academic Press.

Hereu, B., Linares, C., Sala, E., Garrabou, J., GarciaRubies, A., Diaz, D., \& Zabala, M. (2012). Multiple processes regulate long-term population dynamics of sea urchins on Mediterranean rocky reefs. PLoS ONE, 7(5), e36901.

Herrero-Pérezrul, M. D., Ramírez-Ortíz, G., RosalesEstrada, M., \& Reyes-Bonilla, H. (2015). Densidad poblacional y distribución espacial de erizos de mar (Echinodermata: Echinoidea) en la Isla Socorro, Archipiélago de Revillagigedo, México. Revista de Biología Tropical, 63(2).

Hill, S. K., \& Lawrence, J. M. (2003). Habitats and characteristics of the sea urchins Lytechinus variegatus and Arbacia punctulata (Echinodermata) on the Florida Gulf coast shelf. Marine Ecology, 24(1), 15-30.

Irigoyen, A. J. (2009). Undaria pinnatifida en el Golfo Nuevo, Argentina. CENPAT-CONICET: Informe técnico elevado a la Dirección de pesca de la Provincia del Chubut y Ministerio de Ambiente y Control del Desarrollo Sustentable de Chubut.

Irigoyen, A. J., Trobbiani, G., Sgarlatta, M. P., \& Raffo, M. P. (2011). Effects of the alien algae Undaria pinnatifida (Phaeophyceae, Laminariales) on the diversity and abundance of benthic macrofauna in Golfo Nuevo (Patagonia, Argentina): potential implications for local food webs. Biological Invasions, 13, 1521-1532.

Johnson, C. R., \& Mann, K. H. (1982). Adaptations of Strongylocentrotus droebachiensis for survival on barren grounds in Nova Scotia. Echinoderms. Proceedings of the International Echinoderm Conference, Tampa Bay.

Johnson, C. R., Valentine, J. P., \& Pederson, H. G. (2004). A most unusual barrens: Complex interactions between lobsters, sea urchins and algae facilitates spread of an exotic kelp in eastern Tasmania. Proceedings of the $11^{\text {th }}$ Int Echinoderm Conference, Munich.
Kroh, A., Madeira, P., \& Haring, E. (2011). Species distributions: virtual or real - the case of Arbaciella elegans (Echinoidea: Arbaciidae). Journal of Zoological Systematics and Evolutionary Research 50, 99-105.

Labbé-Bellas, R., Cordeiro, C. A., Floeter, S., \& Segal, B. (2016). Sea urchin abundance and habitat relationships in different Brazilian reef types. Regional Studies in Marine Science, 8, 33-40.

Lawrence, J. M. (1975). On the relationships between marine plants and sea urchin. Oceanography and marine biology: an annual review, 13, 213-286.

Levitan, D. R. (1988). Density-dependent size regulation and negative growth in the sea urchin Diadema antillarum Philippi. Oecologia, 76, 621-629.

Levitan, D. R. (1989). Density-dependent size regulation in Diadema antillarum: effects on fecundity and survivorship. Ecology, 70, 1414-1424.

Martín, J. P., \& Cuevas, J. M. (2006). First record of Undaria pinnatifida (Laminariales, Phaeophyta) in Southern Patagonia, Argentina. Biological Invasions, 8, 1399-1402.

Meretta, P. E., Matula, C., \& Casas, G. N. (2012). Occurrence of the alien kelp Undaria pinnatifida (Laminariales, Phaeophyceae) in Mar del Plata, Argentina. BioInvasions Records, 1(1), 59-63.

Morsan, E. M. (2008). Impact on biodiversity of scallop dredging in San Matías Gulf, northern Patagonia (Argentina). Hidrobiologia, 619, 167-180.

Muthiga, N. A., \& Jaccarini, V. (2005). Effects of seasonality and population density on the reproduction of the Indo-Pacific echinoid Echinometra mathaei in Kenyan coral reef lagoons. Marine Biology, 146, 445-453.

Newcombe, E., Cardenas, C., \& Geange, S. (2012). Green sea urchins structure invertebrate and macroalgal communities in the Magellan Strait, southern Chile. Aquatic Biology, 15(2), 135-144.

Ohno, M. (1996). The resource of seaweeds on 21 Century. Tokyo: Midori Press.

Palacín, C., Giribet, G., Carner, S., Dantart, L., \& Turon, X. (1998). Low densities of sea urchins influence the structure of algal assemblages in the western Mediterranean. Journal of Sea Research, 39(3-4), 281-290.

Paredes, R. A. (2010). Erizos de mar: Dinámica poblacional y reclutamiento de larvas dentro de la isla San Cristóbal en el Archipiélago de Galápagos-Ecuador. Universidad San Francisco de Quito, Quito.

Parker, J. D., Burkepile, D. E., \& Hay, M. E. (2006). Opposing effects of native and exotic herbivores on plant invasions. Science 311, 1459-1461. 
Pearse, J. S., \& Hines, A. H. (1987). Long-term population dynamics of sea urchins in a central California kelp forest: rare recruitment and rapid decline. Marine Ecology Progress Series, 39, 275-283.

Raffo, M. P., Irigoyen, A. J., Casas, G. N., \& Schwindt, E. (2012). Efectos del alga exótica Undaria pinnatifida sobre la comunidad de macroalgas bentónicas luego de 15 años de invasión (Golfo Nuevo, Chubut). In H. E. Zaixso, D. G. Gil, M. Varisco \& P. Stoyanoff (Eds.), VIII Jornadas Nacional de Ciencias del Mar. Comodoro Rivadavia.

Rios, C., Mutschke, E., \& Cariceo, Y. (2003). Estructura poblacional de Pseudechinus magellanicus (Philippi1857) en grampones de la macroalga sublitoral Macrocystis pyrifera (L.) C. Agardh en el estrecho de Magallanes, Chile. Anales Instituto Patagonia (Chile), 31, 75-86.

Rivas, A. L., \& Ripa, P. (1989). Variación estacional de la estructura termo-halina de golfo Nuevo, Argentina. Geofisica Internacional, 28(1), 3-24.

Sala, E., Ribes, M., Hereu, B., Zabala, M., Alva, V., Coma, R., \& Garrabou, J. (1998). Temporal variability in abundance of the sea urchins Paracentrotus lividus and Arbacia lixula in the northwestern Mediterranean: comparison between a marine reserve and an unprotected area. Marine Ecology Progress Series, $168,135-145$.

Tegner, M. J., \& Dayton, P. K. (1981). Population structure, recruitment and mortality of two sea urchins (Strongylocentrotusfranciscanus and S. purpuratus) in a kelp forest. Marine Ecology Progress Series, $5,255-268$
Teso, S. V., Bigatti, G., Casas, G. N., Piriz, M. L., \& Penchaszadeh, P. E. (2009). Do native grazers from Patagonia, Argentina consume the invasive kelp Undaria pinnatifida? Revista Museo Argentino de Ciencias Naturales, 11(1), 7-14.

Thiel, M., \& Vásquez, J. A. (2000). Are kelp holdfast islands opn the ocean floor?-Indication for temporarily closed aggregations of peracarid crustaceans. Hydrobiologia, 440, 45-54.

Turon, X., Giribet, S., Lopez, S., \& Palacín, C. (1995). Growth and population structure of Paracentrotus lividus (Echinodermata: Echinoidea) in two contrasting habitats. Marine Ecology Progress Series, 122, 193-204.

Vadas, R. L., Barry, D. S., Beal, B., \& Dowling, T. (2002). Sympatric growth morphs and size bimodality in the green sea urchin (Strongylocentrotus droebachiensis). Ecological Monograph, 72(1), 113-132.

Zaixso, H. E., Boraso de Zaixso, A. L., Pastor de Ward, C. T., Lizarralde, Z., Dadón, J., \& Galván, D. E. (2015). El bentos costero patagónico. In H. E. Zaixso (Ed.), La zona costera patagónica Argentina (Vol. 2). Comodoro Rivadavia, Argentina. Editorial Universitaria Patagónica.

Zaixso, H. E., \& Lizarralde, Z. I. (2000). Distribución de equinodermos en el golfo San José y sur del golfo San Matías (Chubut, Argentina). Revista de Biología Marina y Oceanografia, 35(2), 127-145.

Zaixso, H. E., Lizarralde, Z. I., Pastor, C. T., GomesSimes, E., Romanello, E., \& Pagnoni, G. (1998). Distribución espacial del macrozoobentos submareal del Golfo San José (Chubut, Argentina). Revista de Biología Marina y Oceanografia, 33(1), 43-72. 